\title{
Posterior vertebral column resection as a safe procedure leading to solid bone fusion in metastatic epidural spinal cord compression
}

\author{
Lennart Viezens, MD, ${ }^{1}$ Marc Dreimann, MD, ${ }^{1}$ Sven Oliver Eicker, MD, ${ }^{2}$ Annika Heuer, MD, ${ }^{1}$ \\ Leon-Gordian Koepke, MD, ${ }^{1}$ Malte Mohme, MD, ${ }^{2}$ Theresa Krätzig, MD, ${ }^{2}$ and \\ Martin Stangenberg, MD1
}

${ }^{1}$ Division of Spine Surgery, Department of Trauma and Orthopedic Surgery, and ${ }^{2}$ Department of Neurosurgery, University Medical Center Hamburg-Eppendorf, Hamburg, Germany

\begin{abstract}
OBJECTIVE Cancer is one of the leading causes of death and greatly decreases a patient's quality of life. Vertebral metastases often lead to epidural spinal cord compression (ESCC) requiring surgical therapy. It has previously been shown that in patients with metastatic ESCC (MESCC), a surgical intervention leads to an improved outcome. Although the treatment paradigms in spinal metastases have changed and separation surgery followed by stereotactic radiosurgery is considered the best strategy, there are still cases in which $360^{\circ}$ decompression with stabilization is indicated. In these patients, a proper bone fusion should be the treatment goal to guarantee good clinical results in extended survival times through progressions in oncological therapies. The aim of this study was to examine the safety and feasibility of posterior vertebral column resection (pVCR) in everyday clinical practice, achievement of bone fusion, and midterm outcome in patients with MESCC.
\end{abstract}

METHODS All patients treated with pVCR due to MESCC between 2013 and 2020 were enrolled in this observational single-center study. Demographics, outcome parameters, numeric rating scale (NRS) score, Frankel grade, and Karnofsky Performance Scale (KPS) score were evaluated. Radiological images routinely acquired during follow-up were reviewed and screened for the presence of bone fusion.

RESULTS Sixty-six patients were treated by eight surgeons. The mean follow-up period was $549 \pm 739$ days. At baseline, the average age was $64.4 \pm 10.9$ years. Reported NRS scores (preoperative $6.2 \pm 1.7$ vs postoperative $3.4 \pm 1.6$ ) and segmental kyphosis as measured on sagittal CT images (preoperative $13.5^{\circ} \pm 8.6^{\circ}$ vs postoperative $3.8^{\circ} \pm 5.4^{\circ}$ ) decreased significantly $(p<0.001)$. In only 2 patients $(3 \%)$, the Frankel grade worsened postoperatively, whereas in 12 patients (18.2\%) an improvement was documented. The KPS score remained constant during the observation period (preoperative $73.2 \% \pm 18.2 \%$ vs $78.3 \% \pm 18 \%$ at last follow-up). Bone fusion was observed in 26 patients (86.7\%) receiving CT more than 100 days after the index surgery.

CONCLUSIONS pVCR is a reliable surgical technique in daily clinical practice, which proves to be beneficial in terms of short- as well as midterm outcome, as judged by the KPS and NRS. The overall improvement in the Frankel grade shows patient safety. A bone fusion was observed regularly in oncological patients undergoing pVCR. The authors therefore conclude that pVCR is a safe, fast, and efficient strategy to achieve stability and pain relief by achievement of bone fusion in cancer patients.

https://thejns.org/doi/abs/10.3171/2021.2.FOCUS201087

KEYWORDS spine tumor; tumor decompression; vertebral column resection; oncological spine surgery; spinal cord compression

$\mathrm{B}$ ONE metastases are a frequent and debilitating complication of solid malignancies. ${ }^{1-3}$ The spine is the most frequently affected anatomical site for bone metastases and pathological fractures, predominant- ly found within the thoracic vertebral column. ${ }^{4,5}$ Due to improving imaging techniques and systemic oncological management, an extended life expectancy in tumor patients could be achieved. ${ }^{6-8}$ However, in many cases the

ABBREVIATIONS CUP = cancer of unknown primary origin; ESCC = epidural spinal cord compression; KPS = Karnofsky Performance Scale; MESCC = metastatic ESCC; NRS = numeric rating scale; pVCR = posterior vertebral column resection; SINS = Spine Instability Neoplastic Score.

SUBMITTED December 23, 2020. ACCEPTED February 22, 2021.

INCLUDE WHEN CITING DOI: 10.3171/2021.2.FOCUS201087. 
initial diagnosis of the malignancy is made due to symptoms of the metastatic spread of the primary tumor. Once cancer metastasizes to bone, a cascade of remodeling with increased risk of pathological fractures and pain occurs, severely diminishing the possibility of a cure. ${ }^{9}$ Hence, it is not surprising that the incidence of bone metastases in patients with advanced-stage malignancy can be as high as $90 \% .^{10-14}$

In lytic lesions, structural integrity is often impaired with a high risk of fractures, potentially warranting surgical reconstruction. ${ }^{15,16}$ High doses of opioids are often required to manage the immobilizing pain following pathological fractures or metastatic epidural spinal cord compression (MESCC) due to tumor invasion of the epidural space. MESCC greatly decreases a patient's quality of life by functional and psychological sequelae, disability, and neuropathic pain. ${ }^{17}$ In 35\%-75\% of oncological patients presenting with spinal metastases, motor deficits are already present at diagnosis. ${ }^{8}{ }^{8} 18$ Patchell et al. showed that in cases of MESCC and spinal instability, surgical intervention was associated with prolonged patient mobility and independence, preserving a good quality of life. ${ }^{19}$

Surgical interventions range from minimally invasive to highly sophisticated individualized techniques, and have evolved over the years ${ }^{20}$ from decompressive surgery alone, ${ }^{21}$ to decompressive surgery with posterior stabilization, ${ }^{22}$ to decompressive surgery with posterior and anterior stabilization, ${ }^{23}$ and different techniques of posterior vertebral column resection (pVCR). ${ }^{24-28}$ Once MESCC occurs, even with adept management, treatment usually aims to preserve function rather than reverse neurological deficits. Over the last decade, treatment paradigms in cancer patients with spinal metastases changed in favor of less aggressive surgical procedures such as separation surgery followed by stereotactic radiosurgery, with an excellent progression-free survival. ${ }^{29,30}$

However, tumor destruction of the cortical bone can lead to vertebral collapse, a retropulsion of bone fragments into the epidural space, and a misalignment of the spine, which still requires a $360^{\circ}$ decompression with restoration of spinal alignment. ${ }^{17,25} \mathrm{~A}$ ventral approach to the upper and midthoracic region is particularly challenging for surgeons. In the authors' department the application of a pVCR with $360^{\circ}$ decompression using anterior as well as posterior spondylodesis through a single dorsal approach is frequently used as a feasible surgical procedure in selected patients. ${ }^{24,25,31}$ However, it has not yet been sufficiently investigated whether this procedure is safe in daily clinical practice as opposed to only being effective in the hands of a highly skilled surgeon. Furthermore, controversy continues to exist as to whether a sufficient bone fusion of the operated spine is achievable despite postoperative radiation therapy. ${ }^{32,33} \mathrm{~A}$ sufficient bone fusion is essential after spinal fixation surgery in various spinal diseases with regard to permanent stability and long-lasting pain relief. ${ }^{32,34}$

In this single-center observational study, we aimed to evaluate the safety of this above-described surgical technique in standard clinical care, as well as provide an analysis of objectively measured and individual outcome parameters, such as achievement of physiological sagittal alignment values, patient performance status over time,
Frankel grade, and pain according to the numeric rating scale (NRS). Furthermore, we aimed to evaluate bone fusion on $\mathrm{CT}$ as a surrogate for permanent stability and longlasting pain relief.

\section{Methods \\ Cohort Description}

From 2013 onward, data of all patients receiving a pVCR due to spinal metastasis with ESCC were collected. In December 2020, a retrospective analysis was performed. Baseline data consisted of each patient's age, sex, BMI, and preexisting medical conditions. The Tomita score,,$^{35}$ modified Tokuhashi score, ${ }^{36}$ Spine Instability Neoplastic Score (SINS), ${ }^{37}$ and ESCC score ${ }^{38,39}$ were calculated. The pre- and postoperative spinal Cobb angles for kyphosis were measured using CT scans. Furthermore, shortening due to compression during pVCR was evaluated. Blood loss during the operation, operative duration, in-hospital stay, and status at follow-up visits were documented. At analysis, all available follow-up CT images were screened for bone fusions.

\section{Indication for Surgical Intervention}

pVCR was performed in patients with ESCC grade 2 or 3 presenting with either existing or imminent neurological deficiencies or a high-grade osteolytic destruction that was considered unstable, or because of an interdisciplinary tumor board decision. In preparation for surgery, the Tomita and modified Tokuhashi scores were calculated. The Tomita score ${ }^{35}$ is a patient-centered score suggesting a surgical strategy in relation to the expected survival period. The score gives a recommendation on the radicality of the surgical procedure. The modified Tokuhashi ${ }^{36}$ score also attempts to calculate an approximate life expectancy, ultimately influencing further therapy.

\section{Surgical Technique}

To achieve complete $360^{\circ}$ decompression, instrumentation, and fusion, each operation was performed using a standardized approach by one of eight certified spine surgeons (either a neurosurgeon or orthopedic surgeon) as previously described by Dreimann et al. ${ }^{25}$ The following steps were carried out for $\mathrm{pVCR}$.

After preoperative imaging review and surgical planning, the patient was positioned prone after induction of general anesthesia. The incision was made linearly over the midline and centered on the level of metastasis (the index level), followed by preparation of the paraspinal muscles in the target area. This was followed by instrumentation with pedicle screws, usually 2 vertebral bodies above and 2 below the index vertebra. Depending on bone density, additional cement augmentation of the screws was performed. Subsequently, a temporary rod was inserted on one side before a $360^{\circ}$ decompression with resection of both pedicles and complete resection of the posterior longitudinal ligament was performed. Depending on the preexisting bone impairment, the vertebral body was resected using curettes or a diamond burr. If needed, ligature of the nerve roots of the index segment facilitated wider access, and in our clinical expertise is not noticed 
A

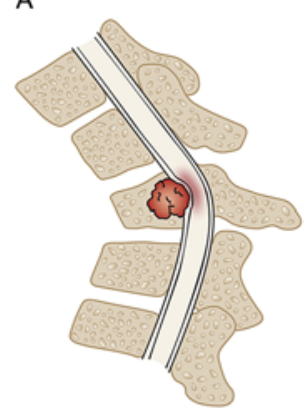

B

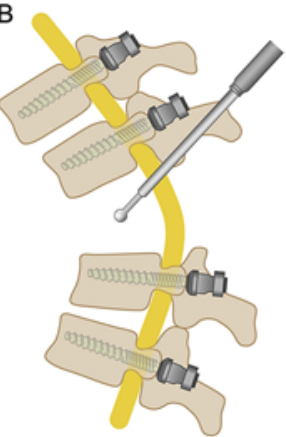

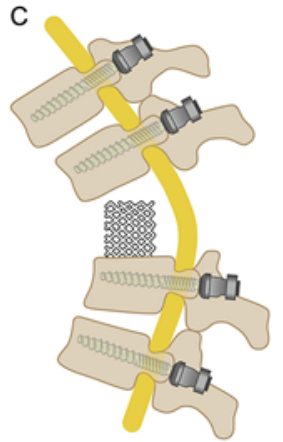

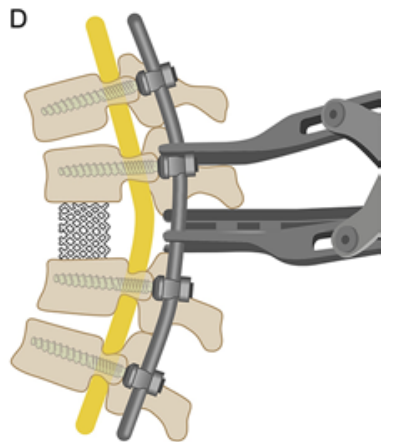

FIG. 1. Schematic diagram of the surgical pVCR technique. A: Pathological fracture with ventral epidural tumor compression and secondary hyperkyphosis leading to ESCC. B: Dorsal instrumentation and $360^{\circ}$ decompression with the diamond burr. C: Insertion of the mesh cages as a ventral hypomochlion. D: Gradual dorsal compression via pre-bent rods leading to a reduction of both length and kyphosis. Copyright Lennart Viezens. Published with permission.

by the patient postoperatively in the thoracic area (T3-12). After successful preparation of the vertebral body and both adjacent intervertebral discs, two fitted titanium mesh cages filled with bone substitute to facilitate fusion were inserted from both sides. To prevent distraction, a rod was utilized unilaterally to achieve stabilization for the course of the operation. The cages were firmly clamped between the adjacent vertebrae via subsequent dorsal compression, during which correction of a hyperkyphotic deformity could be achieved (Fig. 1). Finally, dorsal spondylodesis and the usual stepwise wound closure finalized the procedure. After the operation, monitoring took place on the intermediate care ward.
Radiological control with CT was regularly obtained at the 1st postoperative day. Pain-adapted mobilization was conducted under physiotherapeutic guidance until discharge. Further therapy was determined by interdisciplinary tumor board consensus after receiving the final histopathological report. Postoperative management after discharge included analgetic therapy, functional treatment without peak load exercises, as well as antiresorptive treatment (e.g., bisphosphonates).

\section{Follow-Up}

Follow-up was routinely performed at 3, 6, and 12 months postoperatively and in a 12 -month cycle thereafter.
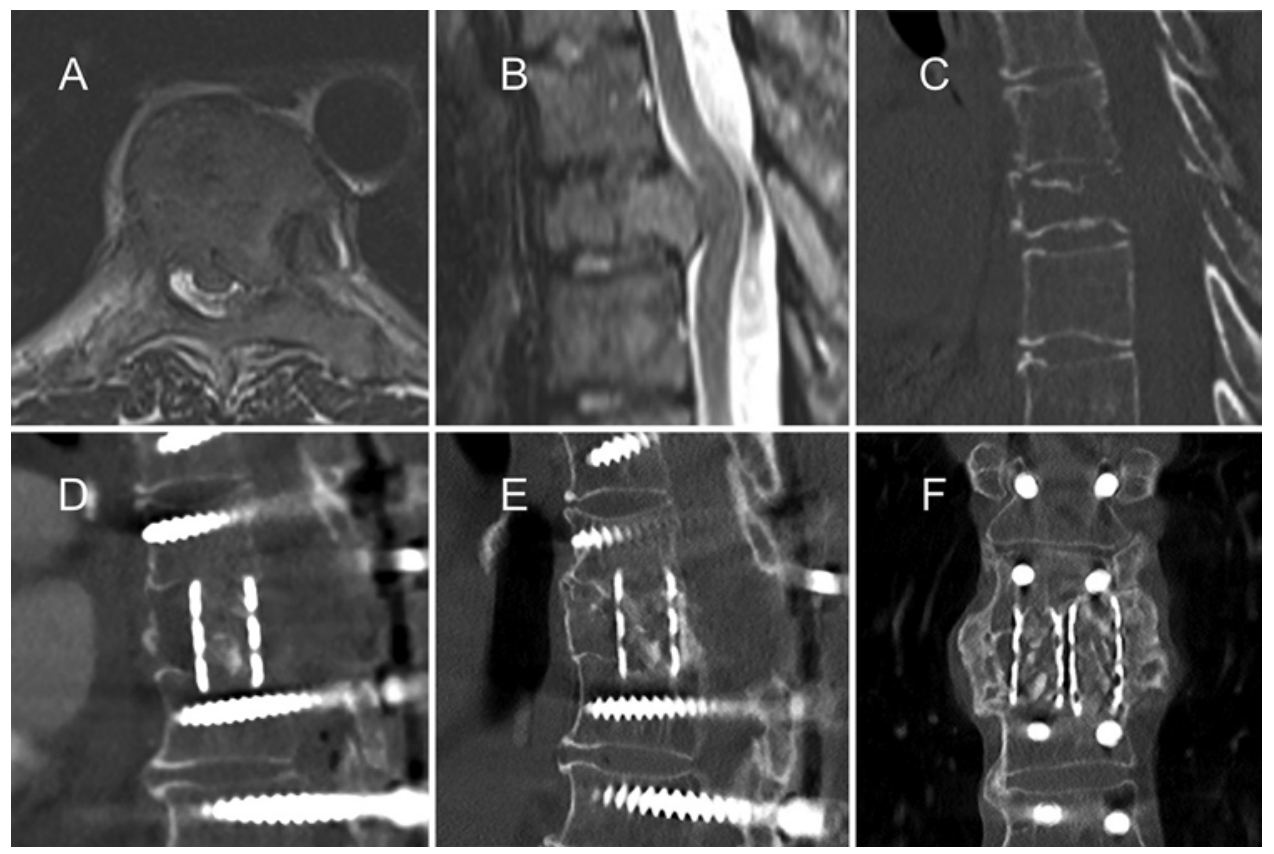

FIG. 2. Example of a patient with MESCC and pVCR of T7. A: Preoperative T2-weighted MR image with ESCC grade 2 in the axial plane. B: Corresponding preoperative T2-weighted MR image in the sagittal plane. C: Sagittal preoperative CT scan showing osteolysis of the vertebral body with destruction of the rear edge. D: Sagittal CT scan on day 1 after pVCR surgery demonstrating one of the titanium mesh cages in the correct position. E: CT scan 9 months after surgery showing bone fusion anterior and posterior to the cage in the sagittal plane. F: Coronal plane CT image 9 months after surgery with good bone fusion lateral to the cages. 
TABLE 1. Tumor scores and histopathological examination

\begin{tabular}{lcc}
\hline \multicolumn{1}{c}{ Variable } & Preop & Postop \\
\hline Mean modified Tokuhashi score \pm SD, points & $9.7 \pm 2.2$ & \\
\hline Mean Tomita score \pm SD, points & $4.4 \pm 1.8$ & \\
\hline Mean SINS \pm SD, points & $11.4 \pm 2.5$ & \\
\hline Mean ESCC \pm SD, grade & $2.7 \pm 0.5$ & \\
\hline Tumor histology, $\mathrm{n}$ & & \\
\hline CUP & 32 & 0 \\
\hline Breast & 13 & 14 \\
\hline Myeloma & 6 & 18 \\
\hline Prostate & 1 & 2 \\
\hline Lung & 3 & 10 \\
\hline Renal cell & 3 & 6 \\
\hline Reticular cell tumor (spleen) & 0 & 1 \\
\hline Sarcoma & 1 & 3 \\
\hline Oropharynx & 1 & 1 \\
\hline Colon & 1 & 1 \\
\hline Rectum & 3 & 3 \\
\hline Endometrium & 0 & 1 \\
\hline Thyroid & 1 & 1 \\
\hline Hemangiopericytoma & 0 & 1 \\
\hline Cholangiocellular & 0 & 1 \\
\hline Auditory canal & 0 & 1 \\
\hline Gastrointestinal stroma tumor & 1 & 1 \\
\hline Melanoma & 0 & 1 \\
\hline
\end{tabular}

The results of the Frankel grade, NRS score, Karnofsky Performance Scale (KPS) score, and radiological parameters were determined pre- and postoperatively as well as during every presentation at our outpatient clinic during follow-up. We used the NRS, an 11-point scale, to objectively measure axial pain experienced by the patient in a reproducible way; KPS score, an 11-point scale describing the functional impairment in everyday life; and the neurological status according to Frankel, ${ }^{40}$ classifying the extent of the neurological/functional deficit into 5 grades.

\section{Radiological Screening}

Conventional radiography was performed at 3,6 , and 12 months postoperatively, or whenever needed due to clinical indication. CT scans were regularly obtained postoperatively for restaging and cancer follow-up care. For objective evaluation, scores in regard to imaging modality were calculated: CT imaging was evaluated using the SINS, and MRI was judged by the ESCC score. ${ }^{37,39}$ The presence of bone fusions was evaluated on CT. Proper fusion was considered to be a continuous bone brace across the index segment on the sagittal or coronal plane (Fig. 2). To determine the original height before pathological fracturing occurred, the combined heights of the vertebral bodies above and below were measured and divided by 2 . After surgery, the height between the two adjacent endplates was measured and the shortening was calculated.

\section{Statistical Analysis}

The study was reported according to the STROBE guidelines $^{41}$ and acknowledged by the ethics committee of the local medical council. Data were evaluated descriptively. For the pre- and postoperatively collected parameters, a statistical evaluation was conducted using the Student t-test (2-sample assuming equal variances) in SPSS (version 20, IBM Corp.). A p value $<0.05$ indicated a statistically significant difference. All values shown are given as mean \pm standard deviation.

\section{Results}

\section{Baseline Characteristics}

During the 7-year period from 2013 to 2020, 66 patients with metastatic spinal disease received pVCR. Of these patients, 36 (54.5\%) were male and the mean age was $64.4 \pm$ 10.9 years. The average BMI was $25.8 \pm 4.3 \mathrm{~kg} / \mathrm{m}^{2}$, and a total of 10 patients $(15.2 \%)$ were obese (defined as BMI > $30 \mathrm{~kg} / \mathrm{m}^{2}$ ). Arterial hypertension was present in $19(28.8 \%)$, and chronic obstructive pulmonary disease or chronic heart disease in 8 patients (12.1\%). Six patients (9.1\%) presented with diabetes mellitus. Forty patients (60.6\%) had a known malignancy before presenting with spinal metastases.

\section{Indication}

pVCR was performed in 9 patients $(13.6 \%)$ with an ESCC score $<2$ due to an interdisciplinary tumor board decision $(\mathrm{ESCC}$ grade $1 \mathrm{a}=2$, grade $1 \mathrm{~b}=4$, grade $1 \mathrm{c}=$ $3)$. In the remaining 38 cases $(57.5 \%)$ with ESCC grade 3 and 19 cases (28.8\%) with ESCC grade 2, the indication for surgery was determined after considering clinical examination and imaging. The mean preoperative SINS was $11.4 \pm 2.5$ points, and the Tomita score was $4.4 \pm 1.8$ points. The mean modified Tokuhashi score was $9.7 \pm 2.2$ points (Table 1).

In 40 patients $(60.6 \%)$ a previous malignancy was known, although it was not always responsible for the metastasis. Six patients $(9.1 \%)$ presented with a previously unknown secondary tumor that caused spinal metastasis. Despite preoperative staging, metastatic disease was due to a cancer of unknown primary origin (CUP) in $32 \mathrm{pa}-$ tients (48.5\%). Histopathological examination detected that the primary tumor was multiple myeloma in 18 patients (27.3\%), breast carcinoma in 14 (21.2\%), lung carcinoma in $10(15.2 \%)$, and renal cell carcinoma in $6(9.1 \%)$. An overview of the preoperative and definitive histopathological diagnosis and preoperative tumor scores can be found in Table 1 .

\section{Surgical Procedure}

Most pVCRs were performed in the upper thoracic region (Table 2), while only 4 lumbar pVCRs were performed. A total of 68 vertebral bodies were replaced, as 2 patients (3.0\%) underwent pVCR at 2 levels. The average instrumentation length was $4.5 \pm 1.5$ segments. The nerve root of the index segment was sacrificed in 51 cases (77.3\%). The postprocedure CT imaging showed that the preoperatively existing kyphosis was significantly corrected from $13.5^{\circ} \pm$ $8.6^{\circ}$ to $3.8^{\circ} \pm 5.4^{\circ}$ (p < 0.001; Table 3$)$. Due to dorsal com- 
TABLE 2. Local distribution of 68 resected vertebrae in 66 patients

\begin{tabular}{cc}
\hline Localization & No. of Cases \\
\hline T1 & 2 \\
\hline T2 & 7 \\
\hline T3 & 11 \\
\hline T4 & 6 \\
\hline T5 & 4 \\
\hline T6 & 8 \\
\hline T7 & 7 \\
\hline T8 & 4 \\
\hline T9 & 2 \\
\hline T10 & 1 \\
\hline T11 & 7 \\
\hline T12 & 5 \\
\hline L1 & 1 \\
\hline L2 & 2 \\
\hline L3 & - \\
\hline L4 & 1 \\
\hline L5 & - \\
\hline
\end{tabular}

pression during the pVCR, segmental shortening of $6.8 \pm$ $5.1 \mathrm{~mm}$ was recorded, which corresponds to a shortening of $35.4 \% \pm 18.6 \%$ within the operated segment. One malpositioned implant was radiologically observed, which did not require surgical intervention. The average intraoperative blood loss was $2007 \pm 1193 \mathrm{ml}$, and the mean surgical duration was $268 \pm 59$ minutes. Cement augmentation of pedicle screws was performed in 7 cases $(10.6 \%)$.

\section{Radiological Screening}

Each available follow-up CT image was screened for implant loosening, local tumor recurrence, and segmental kyphosis. Overall, we observed 1 screw loosening (not requiring revision surgery), no local tumor recurrence, and a slight loss of kyphosis reduction from $3.8^{\circ} \pm 5.4^{\circ}$ immediately postoperatively to $5.6^{\circ} \pm 5.7^{\circ}$ at the last follow-up. The presence of bone fusion was evaluated independently by the first and last author and showed high interrater reliability with only a single controversial case. Bone fusion was detected in 28 patients $(42.4 \%)$. In patients with CT imaging more than 100 days after surgery, we observed a high rate of bone fusion in $26(86.7 \%)$ of 30 cases (Table 3, Fig. 2).

\section{Outcome Parameters}

The in-hospital time was $12.7 \pm 7$ days. The results of the pre- and postoperative Frankel grades showed a postoperative improvement in 12 cases (18.2\%; Table 4). The reported NRS score was $6.2 \pm 1.7$ points preoperatively and decreased significantly to $3.4 \pm 1.6$ points $(\mathrm{p}<0.001)$ at discharge. A further decrease was observed at the last follow-up contact, showing an additional significant reduction of the pain level to $2.2 \pm 2$ points $(\mathrm{p}<0.001)$. The mean preoperative KPS score was $73.2 \% \pm 18.2 \%$, where-
TABLE 3. Surgical and radiological parameters

\begin{tabular}{lcc}
\hline \multicolumn{1}{c}{ Parameter } & Mean \pm SD & p Value \\
\hline Mean duration of surgery \pm SD, mins & $268 \pm 59$ & \\
\hline Mean blood loss \pm SD, $\mathrm{ml}$ & $2007 \pm 1193$ & \\
\hline Mean segmental kyphosis preop \pm SD, ${ }^{\circ}$ & $13.5 \pm 8.6$ & \\
\hline Segmental kyphosis postop \pm SD, $^{\circ}$ & $3.8 \pm 5.4$ & $<0.001$ \\
\hline Segmental kyphosis at last follow-up \pm SD, $^{\circ}$ & $5.6 \pm 5.7$ & $<0.05$ \\
\hline Shortening of vertebral body height \pm SD, $\%$ & $35.4 \pm 18.6$ & \\
\hline Bone fusion at last follow-up & & \\
\hline Anterior & 20 & \\
\hline Posterior & 1 & \\
\hline Anterior \& posterior & 7 & \\
\hline No bone fusion & 38 & \\
\hline Fusion rate after follow-up $\geq 100$ days & $26 / 30,86.7 \%$ & \\
\hline
\end{tabular}

as after an average of $549 \pm 739$ days it increased to $78.3 \%$ $\pm 18 \%(\mathrm{p}=0.06$; Table 5).

Postoperative radiotherapy was performed in 49 patients $(74.2 \%)$, and mean duration until radiation treatment was $27.5 \pm 14$ days after surgery. Overall, 50 patients (75.8\%) received further systemic oncological therapy.

\section{Complications}

Surgery-related and general complications are shown separately in Table 6. Two patients (3.0\%) underwent surgical revision for postoperative neurological deterioration. We treated 3 patients $(4.5 \%)$ for postoperative wound infections. In addition, in 1 case (1.5\%) an intraoperative dural tear occurred, which required suturing and no further surgical intervention thereafter. Due to general complications, 9 patients (13.6\%) developed pneumonia, $6(9.1 \%)$ a postoperative temporary delirium, and $4(6.1 \%)$ suffered from cardiac decompensation. The 30-day mortality rate was $10.6 \%(\mathrm{n}=7)$. During the entire follow-up period, 7 patients $(10.6 \%)$ had to be treated surgically for another metastasis in the spinal column. No revision surgery was necessary due to implant loosening, malpositioning, or local recurrence of tumor growth.

\section{Discussion}

In this study, 66 patients received $\mathrm{pVCR}$ performed by

TABLE 4. Cross-tabulation of pre- and postoperative Frankel grades

\begin{tabular}{ccccccc}
\hline \multirow{2}{*}{$\begin{array}{c}\text { Preop Frankel } \\
\text { Grade }\end{array}$} & A & B & C & D & E & Total \\
\cline { 2 - 5 } A & 0 & 0 & 0 & 0 & 0 & 0 \\
\hline B & 0 & 1 & 0 & 0 & 0 & 1 \\
\hline C & 0 & 0 & 1 & 4 & 0 & 5 \\
\hline D & 0 & 0 & 0 & 0 & 8 & 8 \\
\hline E & 1 & 0 & 0 & 1 & 50 & 52 \\
\hline Total & 1 & 1 & 1 & 5 & 58 & \\
\hline
\end{tabular}


TABLE 5. Clinical outcome parameters

\begin{tabular}{clc}
\hline Parameter & Mean \pm SD & p Value \\
\hline KPS score (\%) & \\
\hline Preop & $73.2 \pm 18.2$ & \\
\hline Postop & $78.3 \pm 18$ & 0.06 \\
\hline NRS score (points) & & \\
\hline Preop & $6.2 \pm 1.7$ & $<0.001$ \\
\hline Postop & $3.4 \pm 1.6$ & $<0.001$ \\
\hline Last follow-up & $2.2 \pm 2$ &
\end{tabular}

eight different spine surgeons at one institution. Indication for surgery was based on an interdisciplinary reproducible approach considering clinical and radiological findings in a preparatory stage. All patients presented with severe back pain and, in most cases, advanced MESCC due to malignancies with epidural tumor growth, as demonstrated by a mean ESCC score of 2.67. In all patients, anterior tumor compression required a $360^{\circ}$ release of the thecal sac. Concomitant spinal instability was reflected in a preoperatively increased SINS of $11.4 \pm 2.5$ points.

A number of scores were developed to identify patients who could possibly benefit from a more radical approach and avoid unnecessary excessive medical treatment. ${ }^{42} \mathrm{We}$ calculated the Tomita score during the preoperative evaluation of our patients. The mean score of 4.4 points corresponds well with the recommendation for intralesional resection, which we achieved by pVCR. ${ }^{43}$ Furthermore, the modified Tokuhashi score showed an average of 9.65 points, predicting a rather low life expectancy of 6-12 months on average. ${ }^{44}$ More encouraging are our results showing a stable KPS score of $78.25 \%$ (normal activity with effort) during a mean follow-up period of 18 months, compared to the initial preoperative score of $73.18 \%$ (care for self, unable to carry on normal activity or to do active work; $\mathrm{p}=0.06$ ). Because the KPS score incorporates a patient's activity status and independency, it offers a good and reproducible quantification of impairment. ${ }^{45}$

Because the presence of metastatic disease in the spine renders all subsequent therapy palliative, there is controversy as to if and which treatment is appropriate, in terms of both patient safety and individual benefit, as well as socioeconomically. We believe the reversal or delay of MESCC is essential to prevent massive functional and psychosocial sequelae and preserve a patient's quality of life.

Given that the vast majority of spinal metastasis is located primarily in the vertebral body, access to the anterior column is necessary for tumor tissue removal and anterior decompression. The previous literature shows that ventral tumor compression should reach a full $360^{\circ}$ decompression for optimal outcome regarding neurological function. ${ }^{39,46}$ Some authors favor an anterior-posterior approach, though long operating times, caused by multistep surgery or inexperienced teams, have been shown to correlate with an increase in postoperative infection and longer hospital stays. $^{43,44,47}$ Extensive decompression requires the resection of the pedicles and parts of the vertebral body, after which the resection of the adjacent intervertebral discs and the insertion of the ventral mesh cages, in the hands of an
TABLE 6. Perioperative complications

\begin{tabular}{llr}
\hline \multicolumn{1}{c}{ Variable } & No. & $\%$ \\
\hline Surgery-related complications & & \\
\hline Implant failure & $1^{*}$ & 1.5 \\
\hline Dural tear & $1^{*}$ & 1.5 \\
\hline Revision for neurological deterioration & 2 & 3.0 \\
\hline Deep surgical site infection & 3 & 4.5 \\
\hline Seroma & $1^{*}$ & 1.5 \\
\hline Implant malpositioning & $1^{*}$ & 1.5 \\
\hline General complications & & \\
\hline Myocardial infarction & 2 & 3.0 \\
\hline Cardiac decompensation & 4 & 6.1 \\
\hline Cardiac reanimation & 1 & 1.5 \\
\hline Pneumonia & 9 & 13.6 \\
\hline Sepsis & 1 & 1.5 \\
\hline Postop delirium & 6 & 9.1 \\
\hline Pulmonary embolism & 1 & 1.5 \\
\hline Urinary tract infection & 1 & 1.5 \\
\hline Atrial fibrillation postop & 1 & 1.5 \\
\hline Deaths (30-day mortality) & 7 & 10.6 \\
\hline
\end{tabular}

${ }^{*}$ Not requiring revision surgery.

experienced spine surgeon, lead to an insignificant extension of the surgical time. Subsequently, pVCR proved to be reliable considering physiological reconstruction and full decompression, while being time-efficient. A combination of reliability in daily clinical practice and proven beneficial results in terms of short- as well as midterm outcome, pVCR shows significant advantages over other techniques.

The distribution of the treated vertebral levels shows that pVCR has a distinct advantage at the upper thoracic spine. Due to the specific anatomical features of this region, an anterior vertebral body replacement is only feasible through an extended ventral approach. Consistent with this idea, in our study most of the interventions were performed in the upper thoracic spine (Table 2). However, we were also able to show that pVCR in the lumbar region as an individual treatment approach, e.g., in conditions following extensive ventral surgery, can also be safely applied.

A primary aspect of the pVCR procedure is, after adequate $360^{\circ}$ decompression, generating appropriate dorsal compression after insertion of the ventral mesh cages to guarantee stability and regain the alignment of the spine. Thus, attention must be paid to the degree of shortening. To prevent severe neurological deficits, it is of utmost importance to follow the one-third rule described by Tomita et al. ${ }^{48}$ Dorsal shortening by up to one-third can be achieved safely, whereas a shortening of up to two-thirds should only be performed with caution. Segmental shortening by more than two-thirds must be strictly avoided. Our results comply with this rule and show average shortening by $35 \%$. Furthermore, in our experience spinal cord distraction or torsion has to be prevented at all costs, and therefore a unilateral rod to achieve sufficient stabilization should be in place during the entire operation. Following those strategic recommendations, pVCR can be safely 
performed without intraoperative neuromonitoring. The two cases with postoperative neurological deterioration as reported above could be traced back to intraoperative distraction while instability was underestimated, and both longitudinal rods were removed.

A similar technique to the one described in this paper was described by Zhou et al. ${ }^{28} \mathrm{~A}$ major difference in regard to our approach was an incomplete decompression of only $270^{\circ}$. We believe that a resection of the posterior longitudinal ligament is imperative to avoid neurological deficits, and therefore a full $360^{\circ}$ decompression should be achieved. Rustagi at al. ${ }^{27}$ described another similar technique but, in most cases, did not use a solid cage as replacement for the vertebral body, but rather polymethylmethacrylate cement. Inserted cement, in contrast to the implantation of bone chip-filled mesh, cannot provide bony ingrowth. Therefore, potentially inferior results in regard to bone fusion can be expected.

In the current literature, there is no definitive recommendation as to whether bone fusion should be a treatment target in patients with metastatic disease, as most patients will not experience implant loosening during their remaining lifespan. ${ }^{49}$ However, as noted by Galgano et al., ${ }^{32}$ due to the refinement of tumor therapies, improved survival times in patients with metastatic cancer can be achieved, and therefore possible implant loosening should be prevented. We were able to demonstrate that 100 days after pVCR bone fusion could be detected in as many as $86.7 \%$ of cases, supporting sufficient stability.

Regarding the tumor entities we treated, a high rate of CUP syndromes going into surgery was observed. This is explained by the fact that, as a level 1 spine center, many patients with newly diagnosed spine metastases were referred from tertiary hospitals for further treatment. The definitive histopathological examinations show that, with the exception of a lower incidence of prostate carcinoma, the distribution is comparable to previously reported causative malignancies for bone metastases. ${ }^{50}$ This can be explained by the common osteoblastic appearance of prostate carcinoma metastases. In lytic lesions, structural integrity is often impaired with a high risk of fragility fractures, potentially warranting surgical reconstruction. ${ }^{15,16}$ Hence, after spinal spread of prostate cancer, the SINS is usually rather low and therefore reconstruction of the anterior column less often necessary.

At the moment, pVCR is regularly conducted using titanium mesh cages and titanium pedicle screws. In the future, it should be examined whether more modern implants such as carbon screws and polyetheretherketone cages could be used to achieve consistently good results in terms of biomechanical outcome and high fusion rates, but also benefit from significant advantages regarding imaging and irradiation. ${ }^{51}$ The limitations of our study are the single-center, noncomparative single-arm design without differentiation of the outcome with respect to the tumor histology, and the absence of a long-term survival outcome.

\section{Conclusions}

This study shows that pVCR can be used as a safe treatment alternative for patients with epidural tumor growth and relevant malalignment due to secondary kyphosis in daily clinical routine with acceptable risk and favorable outcome. Furthermore, at midterm follow-up we were able to show a stable KPS score, significant pain reduction, and preserved neurological function, as well as development of solid bone fusion as a surrogate for stability.

\section{References}

1. Huang JF, Shen J, Li X, et al. Incidence of patients with bone metastases at diagnosis of solid tumors in adults: a large population-based study. Ann Transl Med. 2020;8(7):482.

2. Tahara RK, Brewer TM, Theriault RL, Ueno NT. Bone metastasis of breast cancer. In: Ahmad A, ed. Breast Cancer Metastasis and Drug Resistance: Challenges and Progress. Springer International Publishing; 2019:105-129.

3. Wong SK, Mohamad NV, Giaze TR, et al. Prostate cancer and bone metastases: the underlying mechanisms. Int J Mol Sci. 2019;20(10):2587.

4. Bruno AG, Burkhart K, Allaire B, et al. Spinal loading patterns from biomechanical modeling explain the high incidence of vertebral fractures in the thoracolumbar region. $J$ Bone Miner Res. 2017;32(6):1282-1290.

5. Mundy GR. Metastasis to bone: causes, consequences and therapeutic opportunities. Nat Rev Cancer. 2002;2(8):584-593.

6. Baumann M, Ebert N, Kurth I, et al. What will radiation oncology look like in 2050? A look at a changing professional landscape in Europe and beyond. Mol Oncol. 2020;14(7): $1577-1585$.

7. Lagergren P, Schandl A, Aaronson NK, et al. Cancer survivorship: an integral part of Europe's research agenda. Mol Oncol. 2019;13(3):624-635.

8. Sciubba DM, Petteys RJ, Dekutoski MB, et al. Diagnosis and management of metastatic spine disease. A review. J Neurosurg Spine. 2010;13(1):94-108.

9. Coleman RE. Metastatic bone disease: clinical features, pathophysiology and treatment strategies. Cancer Treat Rev. 2001;27(3):165-176.

10. Lenz M, Freid JR. Metastases to the skeleton, brain and spinal cord from cancer of the breast and the effect of radiotherapy. Ann Surg. 1931;93(1):278-293.

11. Lote K, Walløe A, Bjersand A. Bone metastasis. Prognosis, diagnosis and treatment. Acta Radiol Oncol. 1986;25(4-6): 227-232.

12. Ortiz Gómez JA. The incidence of vertebral body metastases. Int Orthop. 1995;19(5):309-311.

13. Stoll BA, Parbhoo S. Bone Metastasis: Monitoring and Treatment. Raven Press; 1983.

14. Wong DA, Fornasier VL, MacNab I. Spinal metastases: the obvious, the occult, and the impostors. Spine (Phila Pa 1976). 1990;15(1):1-4.

15. St Delank K, Eysel P. Behandlung von Metastasen der Wirbelsäule. In: Peters KM, König DP, eds. Fortbildung Osteologie 1. Vol 1. Steinkopff-Verlag; 2006:27-33.

16. Freeman AK, Sumathi VP, Jeys L. Metastatic tumours of bone. Surgery $(O x f)$. 2015;33(1):34-39.

17. Levack P, Graham J, Collie D, et al. Don't wait for a sensory level-listen to the symptoms: a prospective audit of the delays in diagnosis of malignant cord compression. Clin Oncol (R Coll Radiol). 2002;14(6):472-480.

18. Harel R, Chao S, Krishnaney A, et al. Spine instrumentation failure after spine tumor resection and radiation: comparing conventional radiotherapy with stereotactic radiosurgery outcomes. World Neurosurg. 2010;74(4-5):517-522.

19. Patchell RA, Tibbs PA, Regine WF, et al. Direct decompressive surgical resection in the treatment of spinal cord compression caused by metastatic cancer: a randomised trial. Lancet. 2005;366(9486):643-648. 
20. Steinmetz MP, Mekhail A, Benzel EC. Management of metastatic tumors of the spine: strategies and operative indications. Neurosurg Focus. 2001;11(6):e2.

21. Gilbert RW, Kim JH, Posner JB. Epidural spinal cord compression from metastatic tumor: diagnosis and treatment. Ann Neurol. 1978;3(1):40-51.

22. Quan GMY, Vital JM, Aurouer N, et al. Surgery improves pain, function and quality of life in patients with spinal metastases: a prospective study on 118 patients. Eur Spine J. 2011;20(11):1970-1978.

23. Xu R, Garcés-Ambrossi GL, McGirt MJ, et al. Thoracic vertebrectomy and spinal reconstruction via anterior, posterior, or combined approaches: clinical outcomes in 91 consecutive patients with metastatic spinal tumors. J Neurosurg Spine. 2009;11(3):272-284.

24. Bilsky MH, Boland P, Lis E, et al. Single-stage posterolateral transpedicle approach for spondylectomy, epidural decompression, and circumferential fusion of spinal metastases. Spine (Phila Pa 1976). 2000;25(17):2240-2250.

25. Dreimann M, Hoffmann M, Viezens L, et al. Reducing kyphotic deformity by posterior vertebral column resection with $360^{\circ}$ osteosynthesis in metastatic epidural spinal cord compression (MESCC). Eur Spine J. 2017;26(1):113-131.

26. Eicker SO, Cornelius JF, Steiger HJ, Hänggi D. 360-degree osteosynthesis via a posterolateral transpedicular approach in high-risk patients. Eur Spine J. 2012;21(6):1207-1213.

27. Rustagi T, Mashaly H, Ganguly R, et al. Transpedicular vertebrectomy with circumferential spinal cord decompression and reconstruction for thoracic spine metastasis: a consecutive case series. Spine (Phila Pa 1976). 2020;45(14):E820-E828.

28. Zhou RP, Mummaneni PV, Chen KY, et al. Outcomes of posterior thoracic corpectomies for metastatic spine tumors: an analysis of 90 patients. World Neurosurg. 2019;123:e371-e378.

29. Barzilai O, Fisher CG, Bilsky MH. State of the art treatment of spinal metastatic disease. Neurosurgery. 2018;82(6):757-769.

30. Laufer I, Rubin DG, Lis E, et al. The NOMS framework: approach to the treatment of spinal metastatic tumors. Oncologist. 2013;18(6):744-751.

31. Wang JC, Boland P, Mitra N, et al. Single-stage posterolateral transpedicular approach for resection of epidural metastatic spine tumors involving the vertebral body with circumferential reconstruction: results in 140 patients. Invited submission from the Joint Section Meeting on Disorders of the Spine and Peripheral Nerves, March 2004. J Neurosurg Spine. 2004; 1(3):287-298.

32. Galgano M, Fridley J, Oyelese A, et al. Surgical management of spinal metastases. Expert Rev Anticancer Ther. 2018;18(5): 463-472.

33. Kim TK, Cho W, Youn SM, Chang UK. The effect of perioperative radiation therapy on spinal bone fusion following spine tumor surgery. J Korean Neurosurg Soc. 2016;59(6):597-603.

34. Kim M, Oh SK, Choi I, et al. Clinical outcomes of posterior thoracic cage interbody fusion (PTCIF) to treat trauma and degenerative disease of the thoracic and thoracolumbar junctional spine. J Clin Neurosci. 2019;60:117-123.

35. Tomita K, Kawahara N, Kobayashi T, et al. Surgical strategy for spinal metastases. Spine (Phila Pa 1976). 2001;26(3): 298-306.

36. Tokuhashi $\mathrm{Y}$, Matsuzaki $\mathrm{H}$, Oda $\mathrm{H}$, et al. A revised scoring system for preoperative evaluation of metastatic spine tumor prognosis. Spine (Phila Pa 1976). 2005;30(19):2186-2191.

37. Fisher CG, DiPaola CP, Ryken TC, et al. A novel classification system for spinal instability in neoplastic disease: an evidence-based approach and expert consensus from the Spine Oncology Study Group. Spine (Phila Pa 1976). 2010; 35(22):E1221-E1229.

38. Bilsky MH, Laufer I, Fourney DR, et al. Reliability analysis of the epidural spinal cord compression scale. J Neurosurg Spine. 2010;13(3):324-328.
39. Quraishi NA, Arealis G, Salem KMI, et al. The surgical management of metastatic spinal tumors based on an Epidural Spinal Cord Compression (ESCC) scale. Spine J. 2015; 15(8):1738-1743.

40. Frankel HL, Hancock DO, Hyslop G, et al. The value of postural reduction in the initial management of closed injuries of the spine with paraplegia and tetraplegia. I. Paraplegia. 1969; 7(3):179-192.

41. University of Bern. STROBE guidelines. Accessed March 12 , 2021. https://www.strobe-statement.org/

42. Tokuhashi Y, Uei H, Oshima M, Ajiro Y. Scoring system for prediction of metastatic spine tumor prognosis. World $\mathrm{J} \mathrm{Or}$ thop. 2014;5(3):262-271.

43. Spiessberger A, Arvind V, Gruter B, Cho SK. Thoracolumbar corpectomy/spondylectomy for spinal metastasis: a pooled analysis comparing the outcome of seven different surgical approaches. Eur Spine J. 2020;29(2):248-256.

44. Cheng H, Clymer JW, Po-Han Chen B, et al. Prolonged operative duration is associated with complications: a systematic review and meta-analysis. J Surg Res. 2018;229:134-144.

45. Péus D, Newcomb N, Hofer S. Appraisal of the Karnofsky Performance Status and proposal of a simple algorithmic system for its evaluation. BMC Med Inform Decis Mak. 2013;13(1):72.

46. Quraishi NA, Gokaslan ZL, Boriani S. The surgical management of metastatic epidural compression of the spinal cord. $J$ Bone Joint Surg Br. 2010;92(8):1054-1060.

47. Viezens L, Reer P, Strahl A, et al. Safety and efficacy of single-stage versus 2-stage spinal fusion via posterior instrumentation and anterior thoracoscopy: a retrospective matched-pair cohort study with 247 consecutive patients. World Neurosurg. 2018;109:e739-e747.

48. Tomita K, Kawahara N, Murakami H, Demura S. Total en bloc spondylectomy for spinal tumors: improvement of the technique and its associated basic background. J Orthop Sci. 2006;11(1):3-12.

49. Alamanda VK, Robinson MM, Thompson JC, et al. Necessity of bony fusion after surgical treatment of metastatic spine tumors. J Am Acad Orthop Surg. 2020;28(19):e853-e859.

50. Schaberg J, Gainor BJ. A profile of metastatic carcinoma of the spine. Spine (Phila Pa 1976). 1985;10(1):19-20.

51. Ringel F, Ryang YM, Kirschke JS, et al. Radiolucent carbon fiber-reinforced pedicle screws for treatment of spinal tumors: advantages for radiation planning and follow-up imaging. World Neurosurg. 2017;105:294-301.

\section{Disclosures}

Dr. Dreimann reports being a consultant to Stryker, Medtronic, and Spineart, and receiving royalties from Spineart. Dr. Eicker reports being a consultant for Stryker and Spineart, and receiving royalties from Spineart.

\section{Author Contributions}

Conception and design: Viezens, Dreimann, Stangenberg. Acquisition of data: Viezens, Dreimann, Eicker, Heuer, Stangenberg. Analysis and interpretation of data: Viezens, Koepke, Mohme, Krätzig, Stangenberg. Drafting the article: Viezens, Heuer, Stangenberg. Critically revising the article: Dreimann, Eicker, Heuer, Koepke, Mohme, Krätzig. Reviewed submitted version of manuscript: Viezens, Dreimann, Eicker, Heuer, Koepke, Mohme, Krätzig, Stangenberg. Approved the final version of the manuscript on behalf of all authors: Viezens. Statistical analysis: Viezens, Stangenberg. Administrative/technical/material support: Eicker, Heuer, Koepke. Study supervision: Viezens, Stangenberg.

\section{Correspondence}

Lennart Viezens: University Medical Center Hamburg-Eppendorf, Hamburg, Germany.1.viezens@uke.de. 